\title{
A Contemporary Retrospective Study of Survival in Dogs With Primary Lung Tumors: 40 Cases (2005-2017)
}

\author{
Ruth J. Rose ${ }^{1,2 *}$ and Deanna R. Worley ${ }^{1,2}$ \\ ${ }^{1}$ Department of Clinical Sciences, Colorado State University, Fort Collins, CO, United States, ${ }^{2}$ Flint Animal Cancer Center, \\ Colorado State University, Fort Collins, CO, United States
}

Objective: To report the median survival time in a contemporary cohort of dogs with primary lung tumors and intrathoracic nodal metastasis.

Design: Retrospective Case Series.

Animals (or sample): Dogs with primary lung tumors treated with lung lobectomy and lymph node biopsy.

Procedures: The medical record database at Colorado State University was queried for dogs with primary lung tumors from January 1, 2005 to December 31, 2017. Patients were identified for inclusion if they had lung lobectomy and an intrathoracic lymph node

OPEN ACCESS

Edited by:

Silke Salavati,

University of Edinburgh,

United Kingdom

Reviewed by:

Irina Gramer,

Royal Veterinary College (RVC),

United Kingdom

Spela Bavcar

University of Edinburgh,

United Kingdom

${ }^{*}$ Correspondence:

Ruth J. Rose

ruth.rose@colostate.edu

Specialty section:

This article was submitted to Comparative and Clinical Medicine,

a section of the journal

Frontiers in Veterinary Science

Received: 12 December 2019

Accepted: 16 September 2020

Published: 23 October 2020

Citation:

Rose RJ and Worley DR (2020) A Contemporary Retrospective Study of

Survival in Dogs With Primary Lung Tumors: 40 Cases (2005-2017).

Front. Vet. Sci. 7:519703.

doi: 10.3389/fvets.2020.519703 biopsy performed. The median survival time (MST) for lymph node positive (LN+) and negative dogs (LN-) was calculated as well as the MST in dogs that did or did not receive adjuvant chemotherapy. Differences were compared between groups with significance set at $p<0.05$.

Results: The MST in LN+ dogs $(n=11)$ was 167 days which was not statistically different from $L N-$ dogs $(n=29)$ at 456 days $(p=0.2407)$. No significant difference in the MST in LN+ dogs was identified between dogs that received adjuvant chemotherapy ( $n$ $=4 ; 110$ days) and those that did not receive adjuvant chemotherapy $(n=6 ; 125$ days) ( $p$ $=0.4409$ ). There was no difference in survival time in $\mathrm{LN}$ - dogs receiving chemotherapy ( $n=12$; 335 days) as compared to those $L N-$ dogs $(n=10)$ that did not receive adjuvant chemotherapy (258.5 days; $p=0.6475$ ).

Conclusions and Clinical Relevance: The survival of primary pulmonary neoplasia in dogs with intrathoracic nodal metastasis is longer than previously reported in this contemporary cohort. Chemotherapy did not appear to improve survival in $\mathrm{LN}+$ or $\mathrm{LN}-$ dogs. The combination of tumor size between 100 and $999 \mathrm{~cm}^{3}$ and positive lymph node status significantly reduced survival.

Keywords: survival, metastasis, lymph node, canine, primary lung

\section{INTRODUCTION}

Primary lung tumors in dogs have a relatively low incidence within the pet population occurring in $\sim 2-4$ dogs per ten thousand in the US and UK (1-5). It is a disease mostly of older dogs with an average age of 10-11 years and may be over-represented in the Boxer, Doberman Pinscher, Australian Shepherd, Irish Setter and Bernese Mountain Dog breeds, although this 
finding is not consistent across studies (5-9). Whereas, there is a clear relationship between cigarette smoking and the increased risk of developing lung cancer in people, no apparent environmental factors have been identified in dogs (10-12). The clinical signs in dogs are coughing in 50-93\%, dyspnea, lethargy, anorexia, weight loss, hemoptysis, and lameness secondary to hypertrophic osteopathy or rarely metastatic lytic lesions (6, 9, $13-18)$. In up to $30 \%$ of cases, primary lung tumors are an incidental finding $(6,9,13,16)$.

Carcinomas comprise the majority of primary lung tumors with metastasis occurring via lymphatic pathways or hematogenously and intra-airway metastasis reported for pulmonary sarcoma $(5,7,19,20)$. The most common carcinomas reported are pulmonary papillary or bronchoalveolar carcinoma and adenocarcinoma (differentiated and non-differentiated) $(9,13,21)$. Of the several prognostic indicators of survival identified, lymph node status, whether lymph nodes are positive $(\mathrm{LN}+)$ or negative $(\mathrm{LN}-)$ for metastasis, greatly impacts survival (13, 21-23). The diagnosis of a $\mathrm{LN}+$ primary lung cancer garners a grave prognosis with a median survival of 60 days (Ogilvie $n=$ 12), 26 days (McNeil $n=15$ ), 58 days (Polton $n=16$ ) and more recently, 131 days (Paoloni $n=6$ ) as compared to 285 days for LN- dogs (13, 21-23). In addition, tumor grade, clinical signs at diagnosis, histologic diagnosis, completeness of tumor excision, and the size of the tumor have also been associated with survival $(13,21,24)$. Given the notable difference in survival time when LN metastasis is present, a thoracic computed tomography (CT) study is encouraged as part of the diagnostic work-up for patients with primary lung tumors (25). CT imaging can detect smaller pulmonary parenchymal changes such as intra-parenchymal metastasis compared to radiography as well as identify the presence of tracheobronchial lymphadenopathy (23, 25-27). Tracheobronchial lymphadenopathy strongly associates with lymphatic metastasis and this may inform an owner's decision about whether or not to proceed with thoracic surgery for tumor removal (23). Based upon the literature, the presence of lymph node metastasis suggests a grave prognosis, however, our clinical impression is that these patients live longer following treatment.

Therefore, we performed a retrospective study to report the median survival time of a contemporary cohort of dogs with primary pulmonary neoplasia and intrathoracic nodal metastasis. Our hypotheses were that the median survival of LN+ dogs having primary pulmonary neoplasia would be greater than 60 days and that adjuvant chemotherapy would have no effect on survival in dogs with lymph node metastasis.

\section{MATERIALS AND METHODS}

\section{Case Selection Criteria}

The medical records database at Colorado State University Veterinary Teaching Hospital was queried for all dogs diagnosed with a primary lung neoplasia between January 1, 2005 and December 31, 2017. Cases were included if a histologic diagnosis of primary pulmonary neoplasia was made and a lymph node biopsy with histopathology was performed at the time of surgery. Cases were excluded if histopathology confirmed a diagnosis of histiocytic sarcoma or mesenchymal neoplasia or if a secondary neoplasia was identified during treatment.

\section{Medical Records Review}

For each dog, signalment, weight, sex, spay/neuter status, clinical signs at presentation, CT findings, tumor size by volume, surgical approach, lung lobe(s) removed, histologic diagnosis of primary tumor and lymph node, mitotic index and grade, histologic margins, adjuvant treatment and date of death were noted. Tumor volume was calculated when measurements were available in three dimensions and then categorized as $<100 \mathrm{~cm}^{3}$, $100-999 \mathrm{~cm}^{3}$, or $>1,000 \mathrm{~cm}^{3}$ as previously reported (24). The survival time for each individual was defined as the time from surgery until death from progressive disease. Patients lost to follow-up and those alive at the time of writing were censored at the last known interaction for median survival time (MST) analysis. The MST was calculated using a Kaplan Meier survival analysis for patients with positive lymph node metastasis, negative lymph node metastasis, and lymph node status with or without adjuvant chemotherapy. A Mantel Cox test was used to evaluate differences between survival with significance set at $p$ $<0.05$. A Cox Regression was performed to evaluate multiple variables related to survival time. A student's t test was utilized to assess differences in the age, gender, weight, and mitotic index between LN+ (positive lymph node metastasis) and LN(negative lymph node metastasis) groups. All statistics were performed using commercially available software ${ }^{1,2}$.

\section{RESULTS}

\section{Case Selection Results}

Four hundred and forty-eight cases of primary lung tumors were identified within the database with 98 of these having surgery for tumor removal. Of these 98 cases, 51 (52.04\%) had a lymph node biopsy performed and 47 (47.96\%) did not. Three patients were removed from the lymph node biopsy group because one patient had insufficient information regarding surgery and two others had multiple concurrent cancers resulting in a total of 48 lymph node biopsy patients. An additional five cases were excluded because of the diagnosis of histiocytic sarcoma, one was removed for the diagnosis of myxosarcoma, and two additional cases were removed due to a secondary neoplasia being identified (thymoma). For the patients that had a lymph node biopsy performed, $11(27.50 \%)$ were found to be $\mathrm{LN}+$ for metastasis and 29 (72.50\%) were LN- for metastasis.

\section{Signalment, Clinical Presentation, and CT Results}

$\mathrm{LN}+$ breeds reported were mixed breed $(n=5 ; 45.45 \%)$, Labrador Retriever $(n=2 ; 18.18 \%)$ and one each $(9.09 \%$ per breed) for German Short-haired Point, Australian Shepherd, Pomeranian, Staffordshire Terrier. LN- breeds included mixed breed dogs $(n=8 ; 27.59 \%)$, Labrador Retrievers ( $n=5 ; 17.24 \%)$, Boston Terriers $(n=2 ; 6.90 \%)$, Lhasa Apso $(n=2 ; 6.90 \%)$,

\footnotetext{
${ }^{1}$ GraphPad Prism 7 (GraphPad Software, San Diego, CA).

${ }^{2}$ IBM SPSS Statistics 2019 (Armonk, NY).
} 
TABLE 1 | Presenting clinical signs.

\begin{tabular}{lcc}
\hline & LN+ $(\boldsymbol{n}=\mathbf{1 1})$ & LN- $(\boldsymbol{n}=\mathbf{2 9})$ \\
\hline Clinical sign/complaint & $9(81.82 \%)$ & $19(65.52 \%)$ \\
Coughing & 0 & $1(3.45 \%)$ \\
Wheezing & $1(9.10 \%)$ & $1(3.45 \%)$ \\
Inappetence & $1(9.10 \%)$ & $1(3.45 \%)$ \\
Exercise intolerance & 0 & $2(6.91 \%)$ \\
Lethargy & $1(9.10 \%)$ & 0 \\
Increased respiratory effort & 0 & $2(6.91 \%)$ \\
Gagging/retching & 0 & $1(3.45 \%)$ \\
Vomiting & 0 & $1(3.45 \%)$ \\
Panting & 0 & $1(3.45 \%)$ \\
Lameness & 0 & 0 \\
Weight loss & 0 & $2(6.91 \%)$ \\
Chronic upper respiratory disease & 0 & $1(3.45 \%)$ \\
Pneumonia & 0 & $4(13.79 \%)$ \\
None & & \\
\hline
\end{tabular}

Staffordshire Terriers $(n=2,6.90 \%)$, and one each $(4.45 \%$ per breed) for Airedale, Australian Shepherd, Border Collie, Fox Terrier, Shih Tzu, Toy Poodle, English Setter, Doberman Pinscher, Greyhound, and Jack Russell Terrier. There were 8 (72.72\%) spayed female LN+ dogs, 15 (51.72\%) spayed female LN- dogs, 3 (27.27\%) castrated male LN+ dogs, 13 (44.83\%) LN- castrated male dogs, and 1 intact male dog with a negative LN biopsy (3.45\%). The mean age of $\mathrm{LN}+$ dogs was 11.97 \pm 1.47 years (9.58-14 years) and was $11.30 \pm 1.99$ years for LN- dogs with a range of 7.75-14.92 years. There was no statistical difference in mean age between the $\mathrm{LN}+$ and $\mathrm{LN}-$ $\operatorname{dogs}(p=0.1879)$.

The most common clinical signs/presenting complaint for all dogs in the study was coughing (Table 1). Seven of $11 \mathrm{LN}+$ (63.64\%) dogs had a CT scan performed wherein 3 (42.86\%) were reported to have enlarged lymph nodes, $3(42.86 \%)$ were reported to be normal and neither the report nor the images were available for review for one case (14.29\%). Twenty-three of 29 (19.31\%) LN- dogs had a CT scan and of these, $2(8.70 \%)$ patients were reported to have enlarged lymph nodes, 21 (91.3\%) were reported to be normal. Tumor volume was available for $7 \mathrm{LN}+(63.64 \%)$ cases. Volume was less than $100 \mathrm{~cm}^{3}$ for $0 \mathrm{LN}+$ cases, $>100$ and $<999 \mathrm{~cm}^{3}$ for $6(85.71 \%)$ and $>1000 \mathrm{~cm}^{3}$ for $1(14.28 \%) \mathrm{LN}+$ cases. For LN- cases, tumor volume was available for $14(48.28 \%)$ cases wherein 7 (50.00\%) were $<100 \mathrm{~cm}^{3}$ and 7 (50.00\%) were between 100 and $999 \mathrm{~cm}^{3}$.

\section{Surgical Details}

For primary pulmonary tumor removal, an intercostal approach was performed for 9 (81.82\%) LN+ cases and 27 (93.10\%) LNcases whereas a median sternotomy was performed in $2(18.18 \%)$ $\mathrm{LN}+$ case and $2(6.90 \%) \mathrm{LN}$ - cases. The left cranial lung lobe was removed in $4(36.36 \%) \mathrm{LN}+$ cases and $3(10.34 \%) \mathrm{LN}$ - cases, the left caudal was removed in 5 (45.45\%) LN+ cases and $7(24.14 \%)$ $\mathrm{LN}$ - cases, the right cranial was removed in 2 (18.18\%) LN+ cases and $6(20.69 \%)$ LN- cases, the right middle was removed in 1 (9.10\%) LN+ cases and 5 (17.24\%) LN- cases, the right caudal was removed in 2 (18.18\%) LN+ and 10 (34.48\%) LN- cases, and the accessory lung lobe was removed in $1(9.10 \%) \mathrm{LN}+$ cases and 2 (6.90\%) LN- cases. Eight LN+ (72.72\%) cases had a single lung lobectomy performed and $3(10.34 \%)$ had multiple lobes excised in order for all visible disease to be removed. For LNcases, 24 (82.76\%) had a single lung lobectomy and 5 (17.24) had greater than one lung lobe removed. Of the lymph nodes biopsied in $\mathrm{LN}+$ cases, $4(36.37 \%)$ were noted to be tracheobronchial, 1 (9.10\%) was sternal, 2 (18.18\%) mediastinal, 3 (27.27\%) hilar and $1(9.10 \%)$ was not specifically denoted. Of the LN- cases, 17 (58.26\%) lymph node biopsies were noted to be tracheobronchial, 5 (17.24\%) mediastinal, 7 (24.14\%) hilar and 1 (3.45\%) was not specifically identified. Three (10.34\%) LN- dogs had more than one intrathoracic lymph node biopsied. For LN+ lymph node biopsies, only one lymph node per dog was noted to be biopsied in the records. The type of lymph node biopsy (incisional biopsy vs. excisional biopsy) was not specifically noted in the surgical reports and no tracheobronchial lymph nodes were biopsied from a median sternotomy approach.

\section{Histologic Variables}

A summary of histologic diagnoses, mitotic index (MI), evidence of vascular or lymphatic invasion, grade, and completeness of surgical excision can be found in Table 2. The most common histologic diagnoses for the $\mathrm{LN}+$ and $\mathrm{LN}-$ tumors were bronchoalveolar carcinoma $[\mathrm{LN}+n=4$ (36.36\%); LN- $n=$ $21(72.41 \%)]$, and papillary pulmonary adenocarcinoma [LN+ $n$ $=3(27.27 \%)$; LN $-n=7$ (24.14\%)]. All tumors were identified histologically as primary lung tumors.

The mitotic index was available for $9 \mathrm{LN}+$ cases $(81.82 \%)$ and in $27 \mathrm{LN}$ - cases $(84.38 \%)$. The (MI) was $<10$ for $1(11.11 \%)$ $\mathrm{LN}+$ cases and $>10$ for $8(88.89 \%) \mathrm{LN}+$ cases. For LN- cases, the MI was available for 25 of 29 cases $(86.20 \%)$. The MI was $<10$ for $12(48.00 \%)$ and $>10$ for $13(52.00 \%)$. Four of eleven $(36.36 \%) \mathrm{LN}+$ dogs had evidence of vascular or lymphatic invasion, 1 (9.10\%) had intrabronchial invasion, and there was no comment regarding vascular or lymphatic invasion for 6 (54.54\%). For LN- dogs, 5 (17.24\%) tumors had evidence of vascular or lymphatic invasion, 17 (58.62\%) had none and there was no comment for 7 (24.14\%). Regarding grade, there were $3(27.27 \%)$ grade II pulmonary carcinomas in the LN+ dogs, 1 (691\%) denoted as "high" and no grade provided for 7 (63.64\%). For the LN- tumors, grade I was reported in 3 (10.34\%), grade II was reported in $14(48.28 \%)$ and there was no comment made for $12(42.38 \%)$ tumors. Lung lobectomy surgical margins were reported as complete in 4 (36.36\%) LN+ and 27 (93.10\%) LNdogs, incomplete in $6(54.55 \%) \mathrm{LN}+$ and $0 \mathrm{LN}-$. No comment was made regarding surgical margins in $1(9.10 \%) \mathrm{LN}+$ and 1 (3.45\%) LN- cases and the margin was not inked in one LNcase $(3.45 \%)$.

\section{Adjuvant Treatment}

Four (36.36\%) LN+ dogs received adjuvant MTD (Maximal Tolerated Dose) chemotherapy. Vinorelbine alone was used in 3 patients $(8,4$ and 3 doses) and the fourth received 7 doses of 
TABLE 2 | Histologic findings as related to LN status.

\begin{tabular}{|c|c|c|}
\hline & $\mathrm{LN}+(n=11)$ & $\mathrm{LN}-(n=29)$ \\
\hline \multicolumn{3}{|l|}{ Histologic diagnosis } \\
\hline $\begin{array}{l}\text { Tubulopapillary } \\
\text { adenocarcinoma }\end{array}$ & $1 / 11(9.10 \%)$ & $0 / 29$ \\
\hline $\begin{array}{l}\text { Bronchoalveolar } \\
\text { carcinoma }\end{array}$ & 4/11 (36.36\%) & 21/29 (72.41\%) \\
\hline $\begin{array}{l}\text { Papillary pulmonary } \\
\text { carcinoma }\end{array}$ & 3/11 (27.27\%) & $7 / 29$ (24.14\%) \\
\hline $\begin{array}{l}\text { Adenosquamous } \\
\text { carcinoma }\end{array}$ & 2/11 (18.18\%) & 1/29 (3.45\%) \\
\hline Anaplastic carcinoma & $1 / 11(9.10 \%)$ & $0 / 11$ \\
\hline $\mathrm{Ml}<10$ & $5 / 11(45.45 \%)$ & 12/29 (41.38\%) \\
\hline $\mathrm{Ml}>10$ & 8/11 (72.72\%) & 13/29 (44.83\%) \\
\hline $\begin{array}{l}\text { Vascular/lymphatic } \\
\text { invasion }\end{array}$ & Yes 4/11 (36.36\%) & $\begin{array}{l}\text { Yes } 5 / 29(17.24 \%) \\
\text { No } 17 / 29(58.62 \%\end{array}$ \\
\hline Intrabronchial seeding & $1 / 11(9.10 \%)$ & 0 \\
\hline \multicolumn{3}{|l|}{ Grade } \\
\hline I & 0 & 3/29 (10.34\%) \\
\hline ॥ & 3/11 (27.27\%) & 14/29 (58.62\%) \\
\hline III & 0 & 0 \\
\hline "High" & $1 / 11(9.10 \%)$ & 0 \\
\hline Not reported & 7/11 (63.64\%) & 12/29 (41.38\%) \\
\hline \multicolumn{3}{|l|}{ Margins } \\
\hline Complete & 4/11(36.36\%) & 27/32 (93.10\%) \\
\hline Incomplete & $6 / 11(54.55 \%)$ & 0 \\
\hline Not reported & $1 / 11$ (9.10\%) & 2/29 (6.90\%) \\
\hline
\end{tabular}

vinorelbine, 6 doses of carboplatin and 2 doses of doxorubicin. Changes in chemotherapy protocols were based on evidence of progressive disease. Four (36.36\%) LN+ dogs had surgery alone, one had surgery and prednisone $(3.91 \%)$ and it could not be determined whether or not chemotherapy was administered in 2 dogs (18.18\%).

For LN- dogs, 11/29 (27.59\%) received adjuvant MTD chemotherapy, $12(41.3 .8 \%)$ did not receive chemotherapy and it was not known for $4(13.79 \%)$ cases. Two of $29(6.90 \%)$ LN- dogs received surgery and Prednisone. Vinorelbine was used as a single agent in 7/11 patients (63.64\%); one dog received 5 doses, 3 dogs received 4 doses, and 2 dogs received 8 doses. One of 11 dogs received chlorambucil (9.10\%), one dog received cyclophosphamide $(9.10 \%)$, and one dog $(9.10 \%)$ received piroxicam, doxycycline, and cyclophosphamide for six weeks and then vinorelbine (5 doses). Three (27.27\%) LN+ dogs and $2(6.90 \%)$ LN- dogs were lost to follow-up, and $3(10.34 \%)$ $\mathrm{LN}-$ dogs were still alive at the time of writing.

\section{Survival Analyses}

The MST for LN+ dogs was 167 days, and for LN- dogs the MST was 456 days, which failed to be statistically different ( $p$ $=0.2407$ ) (Figure 1). No significant difference in the MST in $\mathrm{LN}+$ dogs was identified between dogs that received adjuvant chemotherapy ( $n=4 ; 110$ days) and those that did not receive adjuvant chemotherapy $(n=6 ; 125$ days) $(p=0.4409)$ (Figure 2$)$.

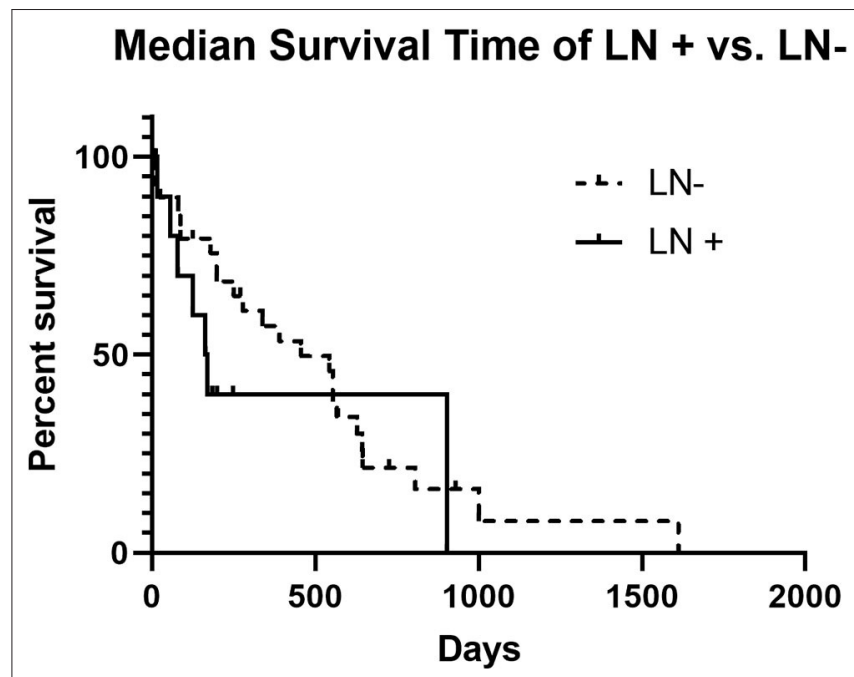

FIGURE 1 | Kaplan-Meier Survival Curve Analysis of LN+ vs. LN- dogs. The median survival time of $\mathrm{LN}+$ dogs was not significantly different than the median survival time for $\mathrm{LN}-$ dogs (167 days vs. 456 days and $p=0.2407$ ).

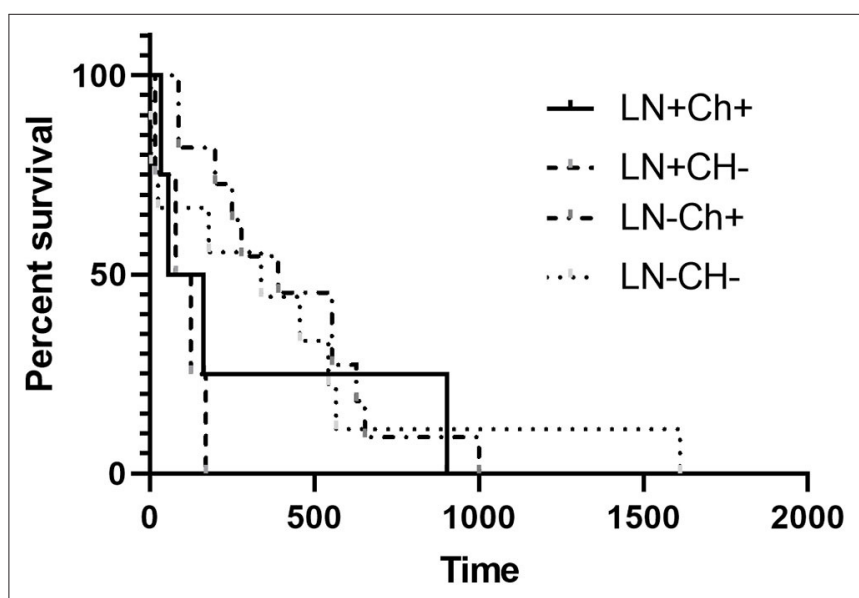

FIGURE 2 | Kaplan-Meier Survival Curve Analysis of the median survival time with or without adjuvant chemotherapy in $L N+$ and $L N-$ dogs. There was no difference in MST for dogs that were $L N+$ or $L N-(P>0.05)$.

There was no difference in survival time in $\mathrm{LN}$ - dogs receiving chemotherapy ( $n=12$; 335 days) as compared to those LNdogs $(n=10)$ that did not receive adjuvant chemotherapy (258.5 days; $p=0.6475$ ). There was no statistical difference in MST for patients that had a MI of $<10$ or $>10$ irrespective of lymph node status $(p=0.1096)$.

The MST between LN+ $(n=4)$ and LN- $(n=7)$ cases where tumor size was $101-999 \mathrm{~cm}^{3}$ was significantly different (110 and 198 days respectively; $p=0.0467$; hazard ratio of 0.5556 with $95 \%$ CI of $0.1626-1.898)$. When evaluating LN- dogs, the MST of dogs with a tumor volume $<100 \mathrm{~cm}^{3}(n=5$; MST 644d) was significantly longer than the MST of LN- dogs with a tumor volume between 100 and $999 \mathrm{~cm}^{3}$ ( $n=7$; MST 198d; $p=0.0232$; hazard ratio of $3.164 ; 95 \%$ CI of $0.0928-1.079)$. No 
other statistical comparisons could be performed on the volume data due to no $\mathrm{LN}+$ cases being in the $<100 \mathrm{~cm}^{3}$ group, and one case in the $\mathrm{LN}+$ and no $\mathrm{LN}-$ cases in the $>1000 \mathrm{~cm}^{3}$ volume category.

The MST for LN+ dogs that received adjuvant MTD chemotherapy, not including Prednisone, $(\mathrm{LN}+\mathrm{CH}+; n=4)$ was 110 days and for those that did not receive adjuvant chemotherapy $(\mathrm{LN}+\mathrm{CH}-; n=4)$, the MST was 102 days wherein there was no significant difference $(p=0.6253)$. For LN- dogs, the MST for dogs that received chemotherapy $(n=11)$ was 390 days and for those that did not $(n=9)$, the MST was 338 days. Patients lost to follow-up and alive at the time of writing were censored prior to survival analysis. There was no difference in the MST for LN- dogs if they received adjuvant chemotherapy $(p=0.7192)$.

\section{DISCUSSION}

In this contemporary cohort of cases, the median survival of LN+ dogs was $\sim 5.5$ months, which is longer than previous reports where the MST ranged from 26-131 days although a direct comparison of MST cannot be performed and one could argue whether or not 131 days as compared to 167 days is clinically relevant $(13,21-23)$. However, this is an important finding to properly inform owners of outcome prior to treatment. The longer median survival time in this $\mathrm{LN}+$ cohort of dogs may be explained by early identification of nodal metastasis on a microscopic level as compared to previous reports. The lack of LN enlargement on pre-operative CT in at least 3 of $11 \mathrm{LN}+$ dogs may support this theory. Similarly, it is being recognized that LN metastasis can occur with no alterations in LN size as is also seen for other canine neoplasms (28). It is strongly recommended that an intrathoracic LN biopsy be performed during lung lobectomy regardless of LN size as determined on CT imaging or intrathoracic assessments, to properly stage the patient and therefore recommend appropriate adjuvant treatment. In this case series, a minimum of 3 patients with metastatic disease would not have been identified had a LN biopsy not been performed based upon the LN size being noted as normal on CT imaging. Retrieval of intrathoracic lymph nodes, particularly the hilar and interlobar lymph nodes, is challenging even with understanding of canine anatomy yet critical for staging (29).

When evaluating the influence of tumor size, we identified that for LN- dogs, a tumor volume $<100 \mathrm{~cm}^{3}$ had a longer survival than dogs where tumor sizes were noted to be between 101 and $999 \mathrm{~cm}^{3}$. As the majority of $\mathrm{LN}+$ cases were within the 101-999 $\mathrm{cm}^{3}$, no other comparisons could be made and not surprisingly, dogs with LN+ and tumor size between 101 and 999 $\mathrm{cm}^{3}$ had a shorter survival time than LN- dogs with a similarly sized tumor.

We failed to reject our hypothesis that there will be no effect on survival with adjuvant chemotherapy for $\mathrm{LN}+$ dogs. Given the low number of patients in this cohort, this data should be interpreted with caution as it may be reflective of type I or type II errors.
The limitations for this study include, low case numbers, possible errors in medical record reporting, histologic grading and re-evaluation of histologic surgical margins were not performed, and the study is retrospective in nature. Therefore, we did not evaluate the effect of tumor grade and completeness of excision on MST. Additionally, there was no standardized method for which intrathoracic lymph nodes were targeted for extirpation in relation to the anatomic lobar site of the excised primary tumor, nor were we able to decipher that the lymph node identified on CT as enlarged was the same lymph node that was biopsied intraoperatively. There was no standardized follow-up schedule utilized, and various chemotherapy agents were administered. A multicenter prospective case-controlled study should be performed to better define and understand the effect of treatment outcomes for dogs with primary pulmonary neoplasia and lymph node metastasis and the efficacy of adjuvant chemotherapy. Although no significant difference with chemotherapy was identified in this retrospective study, there were very few $\mathrm{LN}+$ dogs to compare and this finding should be interpreted with caution.

In conclusion, we have identified a longer survival time in a contemporary series of dogs with primary pulmonary neoplasia with intrathoracic nodal metastasis. Surgical biopsy of intrathoracic lymph nodes during definitive tumor treatment by surgical excision is strongly recommended regardless of LN size on pre-operative imaging for appropriate staging.

\section{DATA AVAILABILITY STATEMENT}

The datasets generated for this study are available on request to the corresponding author.

\section{ETHICS STATEMENT}

Ethical review and approval was not required for the animal study because this was a retrospective study of clinical patients receiving standard of care treatment at the veterinary teaching hospital. Written informed consent for participation was not obtained from the owners because this was a retrospective study of clinical patients receiving standard of care treatment at the veterinary teaching hospital.

\section{AUTHOR CONTRIBUTIONS}

RR and DW contributed equally to the production of this manuscript. Both authors contributed to the article and approved the submitted version.

\section{ACKNOWLEDGMENTS}

The authors would like to thank Dr. Ann Hess for her expertise and contributions regarding statistical analysis and to Dr. Lynn Griffin for re-evaluation of CT images. 


\section{REFERENCES}

1. Dorn CR, Taylor DO, Schneider R, Hibbard HH, Klauber MR. Survey of animal neoplasms in Alameda and Contra Costa Counties, California. II. Cancer morbidity in dogs and cats from Alameda County. J Natl Cancer Inst. (1968) 40:307-18.

2. Moulton JE. Tumors in Domestic Animals. 3rd ed. Berkeley, CA: University of California Press (1990). p. 319.

3. Stunzi H, Head KW, Nielsen SW. Tumours of the lung. Bull World Health Organ. (1974) 50:9-19.

4. Hahn FF, Muggenburg BA, Griffith WC. Primary lung neoplasia in a beagle colony. Vet Pathol. (1996) 33:633-8. doi: 10.1177/030098589603300601

5. Nielsen SW, Horava A. Primary pulmonary tumors of the dog. A report of sixteen cases. Am J Vet Res. (1960) 21:813-30.

6. Brodey RS, Craig PH. Primary pulmonary neoplasms in the dog: a review of 29 cases. J Am Vet Med Assoc. (1965) 147:1628-43.

7. Griffey SM, Kraegel SA, Madewell BR. Rapid detection of Kras gene mutations in canine lung cancer using single-strand conformational polymorphism analysis. Carcinogenesis. (1998) 19:959-63. doi: 10.1093/carcin/19.6.959

8. Meuten DJ. Tumors in Domestic Animals. 4th ed. Ames, IA: Iowa State Press (2002).

9. Ogilvie GK, Haschek WM, Withrow SJ, Richardson RC, Harvey HJ, Henderson RA, et al. Classification of primary lung tumors in dogs: 210 cases (1975-1985). J Am Vet Med Assoc. (1989) 195:106-8.

10. Reif JS, Dunn K, Ogilvie GK, Harris CK. Passive smoking and canine lung cancer risk. Am J Epidemiol. (1992) 135:234-9. doi: 10.1093/oxfordjournals.aje.a116276

11. Bettini G, Morini M, Marconato L, Marcato PS, Zini E. Association between environmental dust exposure and lung cancer in dogs. Vet J. (2010) 186:364-9. doi: 10.1016/j.tvjl.2009.09.004

12. Sabattini S, Mancini FR, Marconato L, Bacci B, Rossi F, Vignoli M, et al. EGFR overexpression in canine primary lung cancer: pathogenetic implications and impact on survival. Vet Comp Oncol. (2014) 12:237-48. doi: $10.1111 /$ vco. 12002

13. McNiel EA, Ogilvie GK, Powers BE, Hutchison JM, Salman MD, Withrow SJ, et al. Evaluation of prognostic factors for dogs with primary lung tumors: 67 cases (1985-1992). J Am Vet Med Assoc. (1997) 211:1422-7.

14. Wouda RM, Miller ME, Chon E, Stein TJ. Clinical effects of vinorelbine administration in the management of various malignant tumor types in dogs: 58 cases (1997-2012). J Am Vet Med Assoc. (2015) 246:1230-7. doi: $10.2460 /$ javma.246.11.1230

15. Rooney JR, II. A primary pulmonary carcinoma in a dog. Cornell Vet. (1956) 46:500-8.

16. Polton G, Finotello R, Sabattini S, Rossi F, Laganga P, Vasconi ME, et al. Survival analysis of dogs with advanced primary lung carcinoma treated by metronomic cyclophosphamide, piroxicam and thalidomide. Vet Comp Oncol. (2018) 16:399-408. doi: 10.1111/vco.12393

17. Marlowe KW, Robat CS, Clarke DM, Taylor A, Touret M, Husbands BD, et al. Primary pulmonary histiocytic sarcoma in dogs: a retrospective analysis of 37 cases (2000-2015). Vet Comp Oncol. (2018) 16:658-63. doi: 10.1111/vco.12437

18. Brodey RS. Hypertrophic osteoarthropathy in the dog: a clinicopathologic survey of 60 cases. J Am Vet Med Assoc. (1971) 159:1242-56.
19. Schaffer PA, Weishaar KM, Han S. Endobronchial metastasis in a dog with sarcoma. J Vet Diagn Invest. (2013) 25:546-50. doi: $10.1177 / 1040638713493779$

20. Moulton JE, von Tscharner C, Schneider R. Classification of lung carcinomas in the $\operatorname{dog}$ and cat. Vet Pathol. (1981) 18:513-28. doi: 10.1177/030098588101800409

21. Ogilvie GK, Weigel RM, Haschek WM, Withrow SJ, Richardson RC, Harvey $\mathrm{HJ}$, et al. Prognostic factors for tumor remission and survival in dogs after surgery for primary lung tumor: 76 cases (1975-1985). J Am Vet Med Assoc. (1989) 195:109-12.

22. Polton GA, Brearley MJ, Powell SM, Burton CA. Impact of primary tumour stage on survival in dogs with solitary lung tumours. J Small Anim Pract. (2008) 49:66-71. doi: 10.1111/j.1748-5827.2007.00403.x

23. Paoloni MC, Adams WM, Dubielzig RR, Kurzman I, Vail DM, Hardie RJ. Comparison of results of computed tomography and radiography with histopathologic findings in tracheobronchial lymph nodes in dogs with primary lung tumors: 14 cases (1999-2002). J Am Vet Med Assoc. (2006) 228:1718-22. doi: 10.2460/javma.228.11.1718

24. Mehlhaff CJ, Leifer CE, Patnaik AK, Schwarz PD. Surgical-treatment of primary pulmonary neoplasia in 15 dogs. J Am Anim Hosp Assoc. (1984) 20:799-803.

25. Ballegeer EA, Adams WW, Dubielzig RR, Paoloni MC, Klauer JM, Keuler NS. Computed tomography characteristics of canine tracheobronchial lymph node metastasis. Vet Radiol Ultrasound. (2010) 51:397-403. doi: 10.1111/j.1740-8261.2010.01675.x

26. Marolf AJ, Gibbons DS, Podell BK, Park RD. Computed tomographic appearance of primary lung tumors in dogs. Vet Radiol Ultrasound. (2011) 52:168-72. doi: 10.1111/j.1740-8261.2010.01759.x

27. Alexander K, Joly H, Blond L, D’Anjou M-A, Nadeau M-E, Olive J, et al. A comparison of computed tomography, computed radiography, and film-screen radiography for the detection of canine pulmonary nodules. Vet Radiol Ultrasound. (2012) 53:258-65. doi: 10.1111/j.1740-8261.2012.0 1924.x

28. Mutz ML, Boudreaux BB, Royal A, Merchant S, Pucheu-Haston C, Griffith EH, et al. Cytologic comparison of the percentage of mast cells in lymph node aspirate samples from clinically normal dogs vs. dogs with allergic dermatologic disease and dogs with cutaneous mast cell tumors. J Am Vet Med Assoc. (2017) 251:421-8. doi: 10.2460/javma.251. 4.421

29. Tuohy JL, Worley DR. Pulmonary lymph node charting in normal dogs with blue dye and scintigraphic lymphatic mapping. Res Vet Sci. (2014) 97:148-55. doi: $10.1016 /$ j.rvsc.2014.07.002

Conflict of Interest: The authors declare that the research was conducted in the absence of any commercial or financial relationships that could be construed as a potential conflict of interest.

Copyright (c) 2020 Rose and Worley. This is an open-access article distributed under the terms of the Creative Commons Attribution License (CC BY). The use, distribution or reproduction in other forums is permitted, provided the original author(s) and the copyright owner(s) are credited and that the original publication in this journal is cited, in accordance with accepted academic practice. No use, distribution or reproduction is permitted which does not comply with these terms. 